\title{
Low temperature CMOS-compatible JFET's
}

\section{J. Vollrath}

Solid State Electronics Laboratory, Technical University of Darmstadt, Schloßgartenstrasse 8 , 64289 Darmstadt, Germany

\begin{abstract}
JFETs used as input transistors for CMOS preamplifiers can improve the input sensitivity due to their lower 1 /f noise. Cryogenic JFET's can be realized by increasing the channel doping concentration to prevent carrier freeze-out. This paper deals with the properties of fabricated cryogenic, CMOS compatible JFET's. Process parameters for a cryogenic JFET technology are presented. Static measurements of these JFET's between room temperature and $4 \mathrm{~K}$ made it possible to extract JFET model parameters $V_{p}$ and $\beta$ for circuit simulations. These measurement results are compared with the temperature dependence of mobility and ionisation.

Noise parameters could be measured at different temperatures with various geometries and doping levels of JFET's.
\end{abstract}

\section{INTRODUCTION}

Infrared detectors, sensors in space and basic physical research experiments operate at very low temperatures /1/. It is convenient to minimize signal loss by placing signal processing electronics operated at low temperatures near the sensors. Cryogenic CMOS-technologies were developed to satisfy these needs $/ 2,3 /$. Problems for small signal detectors arise due to the $1 / \mathrm{f}$ noise level of CMOS-preamplifiers. Since JFET's show lower 1/f noise than CMOS devices it should be possible to use JFETs to improve circuit performance.

At low temperatures JFET's show carrier freeze-out of the channel doping. This can be overcome. Measurements and theory show lower carrier freeze-out with higher channel doping concentrations, as the ionization energy depends on the doping concentration $/ 4 /$. An equation for the ionization energy is given by $/ 5 /$ :

$$
E_{a}=0.0438 \mathrm{eV}-4.08 \cdot 10^{-8} \mathrm{eVcm}\left(N_{A}\right)^{\frac{1}{3}}
$$

Increasing the channel doping concentration $N_{A}$ to $1 * 10^{18} \mathrm{~cm}^{-3}$ minimizes the ionization energy $E_{a}$. No carrier freeze-out at lower temperatures will occur. Special CMOS-compatible JFET's for low-temperature operation can be fabricated $/ 6 /$. 


\section{PROCESS PARAMETERS}

Process parameters can be determined if channel doping concentration $N_{A}$ and channel depth $a$ is known. A breakdown voltage of 10 volts limits the maximum channel doping. To achieve a reasonable pinch-off voltage $V_{P}$ it is necessary to realize a small channel depth a.

$$
V_{P}=\frac{q N_{A} a^{2}}{2 \epsilon_{s}}
$$

$q$ is the charge of an electron and $\epsilon_{s}$ the dielectric constant of silicon. A channel doping of $1 \cdot 10^{18} \mathrm{~cm}^{-3}$ and a pinch-off-voltage of $1 \mathrm{~V}$ leads to a channel depth of $35 \mathrm{~nm}$.

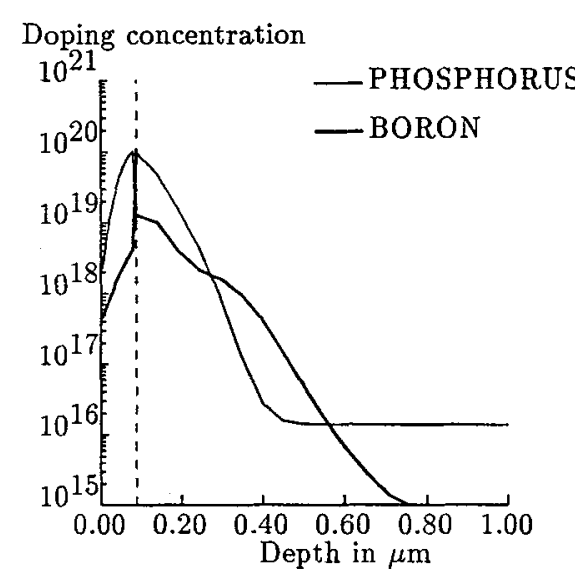

Figure 1: Doping profile
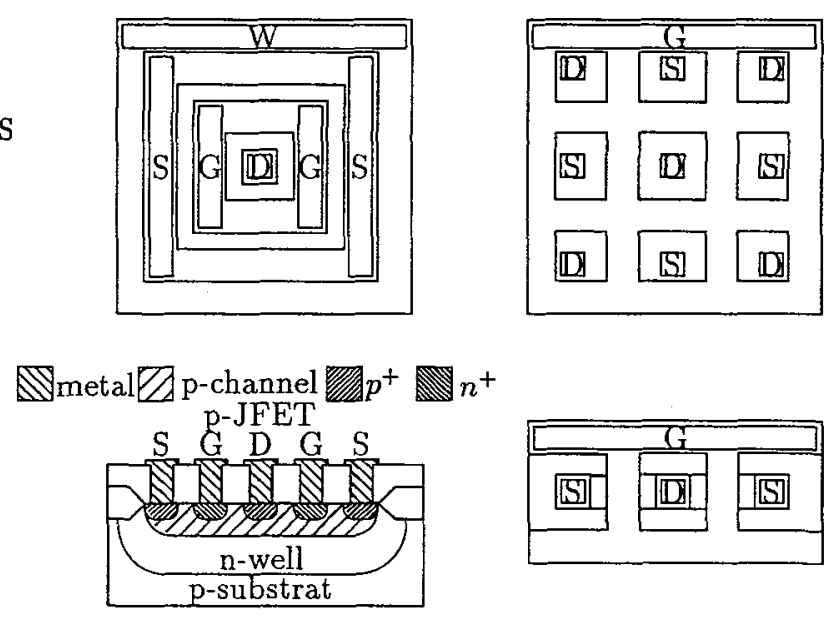

Figure 2: Crossection and geometry of JFET's

CMOS-technology provides drain and source implants which can be used for the gate of the JFET. An additional channel implantation is necessary to control the electrical parameters of the JFET. Process parameters for the channel implantation could be found by ICECREM and SPICES simulations. To achieve a maximum channel doping with a small channel depth, gate and channel should be implanted with a minimal implantation energy and a short annealing time. The resulting doping profile is shown in figure 1. A $90 \mathrm{~nm}$ stray oxide was deposited by dry oxidation. A dose of $1 \cdot 10^{15} \mathrm{~cm}^{-2}$ phosphorus was implanted with $70 \mathrm{keV}$ for the gate. The channel was realized by implanting $2 \cdot 10^{14} \mathrm{~cm}^{-2}$ boron with $40 \mathrm{keV}$. Annealing at $900^{\circ} \mathrm{C}$ for 20 min activated the dopants.

To develop cryogenic JFET's a test chip was designed. Figure 2 shows a crossection and three basic layouts of the JFET's. An ring-JFET, where the drain is surounded by gate and by source brings the advantage of a small gate input capacitance. A checkboard structure provides a large width $\mathrm{W}$ with a minimum area needed for the transistor. Also a simple standard design was made. The n-well of a standard CMOS-process forms the isolation and the second gate of the ring-JFET. These designs were realized with different $\mathrm{W} / \mathrm{L}$ ratios, to compare the dependence between electrical properties and layout. 


\section{THEORIE AND MEASUREMENTS}

A simple JFET-model includes the threshold-voltage $V_{T O}$, the transconductance $\beta$ and the output conductance $\lambda$.

$$
I_{D S}= \begin{cases}0 & : V_{G S}-V_{T O} \geq 0 \\ \beta\left(V_{G S}-V_{T O}\right)^{2}\left(1+\lambda \cdot V_{D S}\right) & : 0>V_{D S}>V_{G S}-V_{T O} \\ \beta V_{D S}\left[2\left(V_{G S}-V_{T O}\right)-V_{D S}\right]\left(1+\lambda \cdot V_{D S}\right) & : 0>V_{G S}-V_{T O} \geq V_{D S}\end{cases}
$$

where

$$
\beta=\frac{I_{p}}{V_{p}^{2}}=\frac{q^{2} \mu_{p} N_{A}^{2} W a^{3}}{\epsilon_{s} L}\left(\frac{2 \epsilon_{s}}{q N_{A} a^{2}}\right)^{2}=\frac{4 W \mu_{p} \epsilon_{s}}{a L} \quad \text { and } \quad V_{T o}=V_{b i}-V_{p}=V_{b i}-\frac{q N_{A} a^{2}}{2 \epsilon_{s}}
$$

The temperature dependence of ionized dopants $N_{A}$, mobilty $\mu_{p}$ and build-in-voltage $V_{b i}$ determine the temperature behaviour of the model parameters $\beta$ and $V_{T O}$.

Lengeler shows in $/ 7 /$ the equations for the number of free electrons and the fermi level. Similar expressions can be found for holes.

$\begin{array}{ccc}\text { freeze-out region } & \text { extrinsic } & \text { intrinsic } \\ \text { compensated } & \text { region } & \text { region }\end{array}$

$$
\begin{aligned}
& N_{D} \geq N_{A} \geq \frac{1}{2} N_{C} e^{-\frac{E_{C}-E_{D}}{k T}} \\
& k T>\frac{E_{C}-E_{D}}{\ln \left(\sqrt{\frac{N_{C}}{2 N_{A}}} \frac{N_{D}-N_{A}}{N_{A}}\right)} \\
& k T<\frac{E_{C}-E_{D}}{\ln \frac{N_{C}}{2 N_{D}}} \\
& n=\frac{N_{D}-N_{A}}{2 N_{A}} N_{C} e^{-\frac{E_{C}-E_{D}}{k T}} \\
& n=\sqrt{\frac{N_{D} N_{C}}{2}} e^{-\frac{E_{C}-E_{D}}{2 k T}} \\
& \frac{E_{C}-E_{D}}{\ln \frac{N_{C}}{2 N_{D}}}<k T<\frac{E_{C}-E_{V}}{\ln \frac{N_{C} N_{V}}{N_{D}{ }^{2}}} \quad \frac{E_{C}-E_{V}}{\ln \frac{N_{C} N_{V}}{N_{D}{ }^{2}}}<k T \\
& n=N_{D} \\
& n=n_{i} \\
& E_{F}=E_{C}-E_{D}+k T \ln \frac{N_{D}-N_{A}}{2 N_{A}} \quad E_{F}=E_{C}-\frac{E_{D}}{2}+\frac{k T}{2} \ln \frac{N_{D}}{2 N_{C}} \\
& E_{F}=E_{C}+k T \ln \frac{N_{0}}{N_{C}} \\
& E_{F}=E_{F i}
\end{aligned}
$$

$N_{C}$ and $N_{V}$ are the effective density of states. Figure 3 shows the calculated temperature dependence of the number of free carriers. At high doping concentration the number of free carriers is proportional to $T^{\frac{3}{4}}$. Lower doping concentrations lead to a steep decrease of free carriers with lower temperatures.

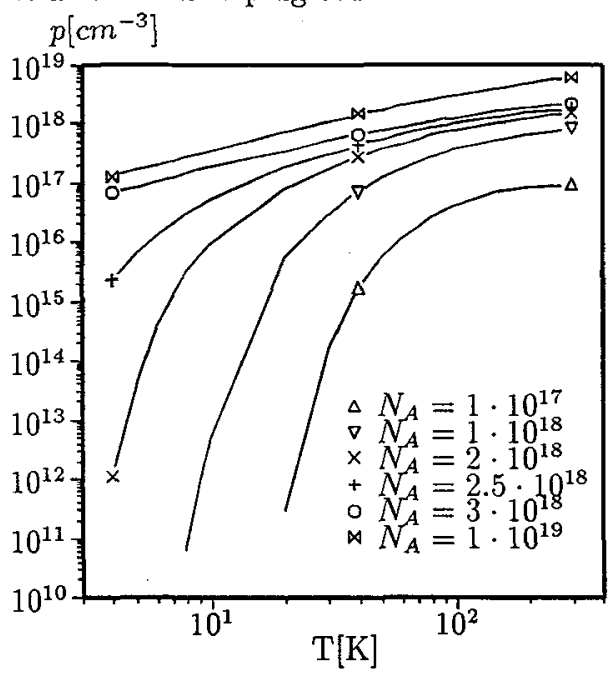

Figure 3: Ionized dopants

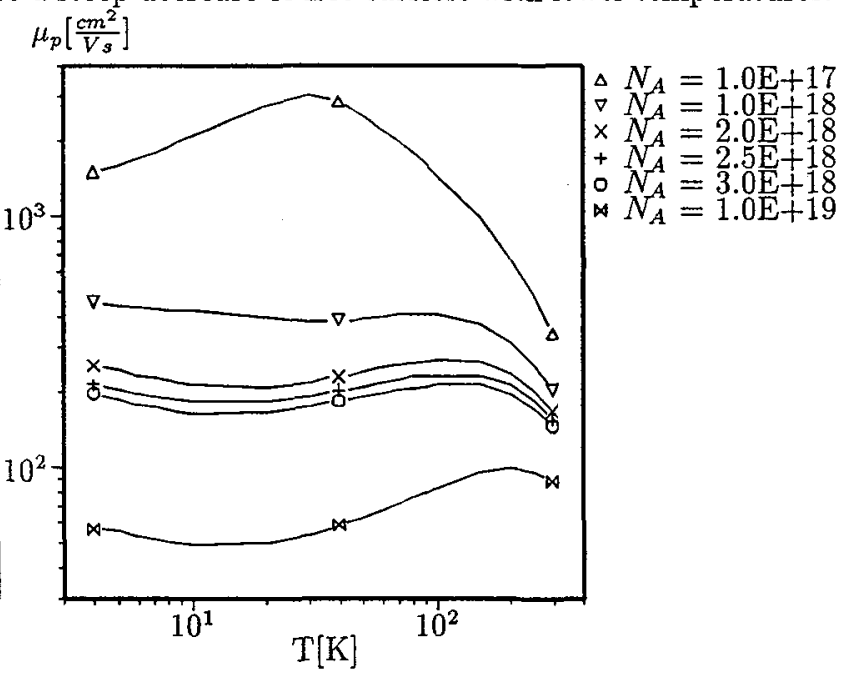

Figure 4: Mobility $\mu_{p}$

Dorkel and Leturcq developed in /8/ an model of the mobility $\mu_{\mathrm{p}}$. Results of the modeling are shown in figure 4 . The mobility has a maximum. The position of the maximum depends on the 
doping concentration. Higher doping concentrations shift the maximum to higher temperatures. Carrier concentration and mobility are compared with the behavior of $\beta$ and $V_{T O}$. Transfer and output characteristics at different temperatures of the above mentioned transistors provided the database for automated model parameter extraction of $\beta$ and $V_{T O}$ (figure 5). $\beta$ decreases from room temperature to $4 \mathrm{~K}$ by a factor of 10000 , but there is still a voltage amplification of about 10 at 4 $\mathrm{K}$. This behavior can be basically explained by the decrease of the number of free carriers. Lower channel concentrations due to the gaussian doping profile in the channel, lead to carrier freeze-out in the lower doped region of the channel and reduce the channel depth a. It is difficult to calculate the physical model parameters. A mathematical approach was chosen.

$$
\beta=\left(\frac{3.84 \frac{p A}{V^{2} K}}{T^{-5.5}+2.25 p K^{-1} T^{86.5 m}}+23.3 \mu \frac{A}{V^{2} K} T\right) \frac{W}{L}
$$

The derivation of the modelling to measurements is less than 10 percent.

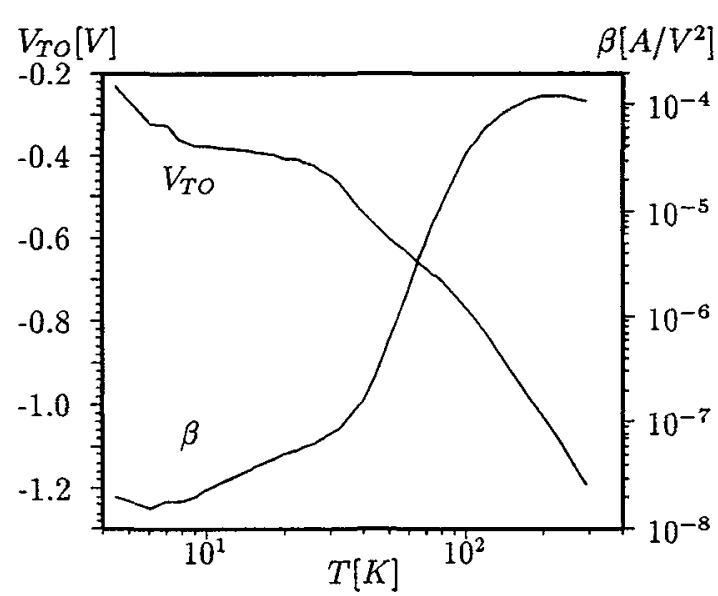

Figure 5:Temperature dependence of $V_{p}$ and $\beta$

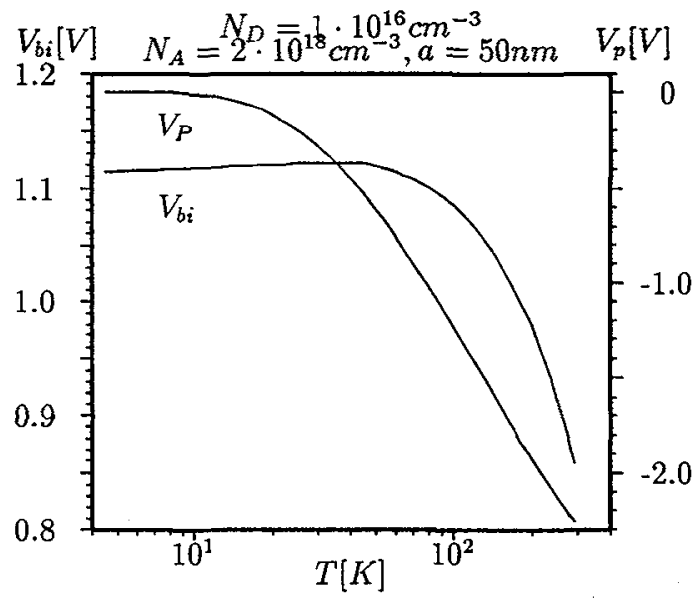

Figure 6:Temperature dependence of $V_{p}$ and $V_{b i}$

Threshold voltage $V_{\text {TO }}$ has two components: pinch-off-voltage $V_{P}$, due to the dopants in the space charge region, and the built-in-voltage $V_{b i}$, due to the different doping concentrations in the channel and the gate. $V_{b i}$ increases and $V_{P}$ decreases with lower temperatures. Since it is difficult to determine the correct doping density $N_{A}$ and channel depth a a mathematical model was chosen.

$$
V_{\text {TO }}=-97 m V \cdot\left(T K^{-1}\right)^{0.476}-78.5 m V\left(T K^{-1}\right)^{-0.57}
$$

Figure 7 shows some measured and modeled transfer characteristics at different temperatures. The agreement is quiet good. 


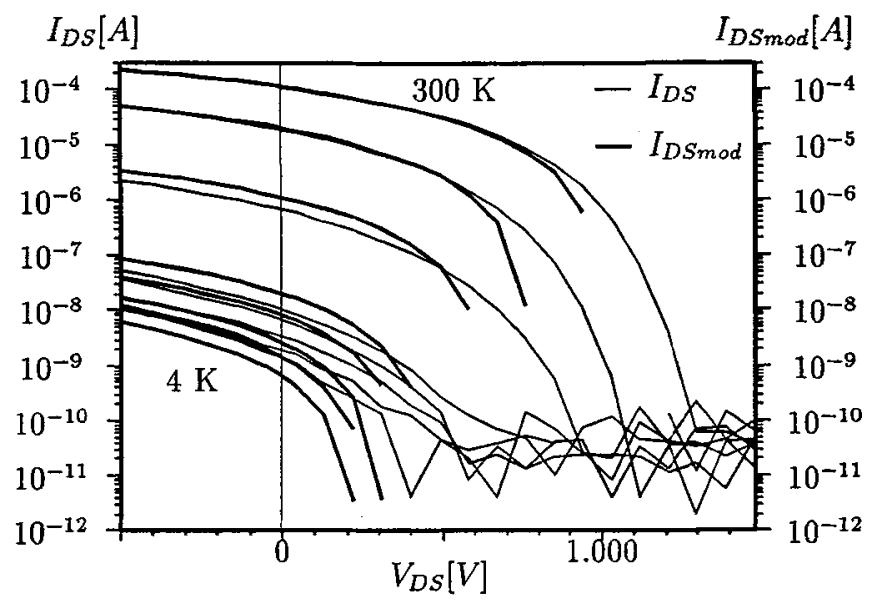

Figure 7: Transfer characteristics at different temperatures

\section{NOISE MEASUREMENTS}

Noise measurements with three different cryogenic JFET's were made (table 1).

\begin{tabular}{|c|c|c|c|c|c|c|c|}
\hline $\begin{array}{c}\mathrm{T} \\
{[K]}\end{array}$ & $\mathrm{W} / \mathrm{L}$ & $\begin{array}{c}\overline{i_{D S}}(1 H z) \\
{[d B A / \sqrt{H z}]}\end{array}$ & $\begin{array}{c}I_{D S} \\
{[A]}\end{array}$ & $\begin{array}{c}K_{F} \\
{[A]}\end{array}$ & $\begin{array}{c}g_{m} \\
{[S]}\end{array}$ & $\begin{array}{c}A_{F} \\
{\left[V^{2}\right]}\end{array}$ & $\begin{array}{c}\overline{e_{n}}(1 \mathrm{~Hz}) \\
{[V / \sqrt{H z}]}\end{array}$ \\
\hline 293 & $40 / 4$ & -201 & $300 \mathrm{n}$ & $2.6 \cdot 10^{-14}$ & $6.7 \mu$ & $1.8 \cdot 10^{-10}$ & $13 \mu$ \\
\hline 293 & $40 / 4$ & -185 & $10 \mu$ & $3.1 \cdot 10^{-14}$ & $75 \mu$ & $5.6 \cdot 10^{-11}$ & $7.5 \mu$ \\
\hline 293 & $40 / 4$ & -178 & $100 \mu$ & $1.6 \cdot 10^{-14}$ & $250 \mu$ & $2.5 \cdot 10^{-11}$ & $5 \mu$ \\
\hline 293 & $20 / 4$ & -178 & $100 \mu$ & $1.6 \cdot 10^{-14}$ & $125 \mu$ & $1.0 \cdot 10^{-10}$ & $10 \mu$ \\
\hline 293 & $20 / 4$ & -188 & $10 \mu$ & $1.6 \cdot 10^{-14}$ & $44 \mu$ & $8.2 \cdot 10^{-11}$ & $9 \mu$ \\
\hline 77 & $40 / 4$ & -228 & $1 \mu$ & $1.6 \cdot 10^{-17}$ & $6 \mu$ & $4.4 \cdot 10^{-13}$ & $664 \mathrm{n}$ \\
\hline 77 & $40 / 4$ & -214 & $3 \mu$ & $1.3 \cdot 10^{-16}$ & $11 \mu$ & $3.3 \cdot 10^{-12}$ & $1.8 \mu$ \\
\hline 77 & $2425 / 5$ & -188 & $100 \mu$ & $1.5 \cdot 10^{-15}$ & $436 \mu$ & $8.3 \cdot 10^{-13}$ & $913 \mathrm{n}$ \\
\hline 77 & $2425 / 5$ & -191 & $30 \mu$ & $2.6 \cdot 10^{-15}$ & $196 \mu$ & $2.1 \cdot 10^{-12}$ & $1.4 \mu$ \\
\hline 77 & $2425 / 5$ & -193 & $3 \mu$ & $1.7 \cdot 10^{-14}$ & $42 \mu$ & $2.8 \cdot 10^{-11}$ & $5.3 \mu$ \\
\hline 77 & $2425 / 5$ & -190 & $1 \mu$ & $1.0 \cdot 10^{-13}$ & $19 \mu$ & $2.7 \cdot 10^{-10}$ & $16 \mu$ \\
\hline 4 & $40 / 4$ & -230 & $30 \mathrm{n}$ & $3.3 \cdot 10^{-16}$ & $57 \mathrm{n}$ & $3.1 \cdot 10^{-9}$ & $55 \mu$ \\
\hline 4 & $2425 / 5$ & -212 & $226 \mathrm{n}$ & $2.8 \cdot 10^{-15}$ & $410 \mathrm{n}$ & $3.9 \cdot 10^{-9}$ & $62 \mu$ \\
\hline 4 & $2425 / 5$ & -228 & $30 \mathrm{n}$ & $5.2 \cdot 10^{-16}$ & $97 \mathrm{n}$ & $1.6 \cdot 10^{-9}$ & $40 \mu$ \\
\hline
\end{tabular}

Table 1: Noise parameters of cryogenic p-channel JFETs for different temperatures

The equivalent input voltage $\overline{e_{n}}$ decreases with a higher current $I_{D S} . \overline{e_{n}}$ is very high for these JFET's and equal compared to MOSFET's. At $77 \mathrm{~K}$ the noise decreases and the noise data at $77 \mathrm{~K}$ show nearly no dependence on $\mathrm{W}$ and L. Detailed measurements showed high gate leakage currents. Noise measurements on a chip with a lower channel doping concentration showed also such high noise levels. Probably problems with the fabrication process lead to high leakage currents, due to the metallization process. 


\section{CONCLUSION}

A special fabrication process leads to CMOS compatible JFET's. Normal operation at cryogenic temperatures was measured. Mathematical model parameters for $\beta$ and $V_{T O}$ could be obtained. Noise levels of fabricated devices were comparable to CMOS devices, due to high reverse gatedrain currents. A different metallization process with a diffusion barrier will probably overcome this problem. A new batch of chips is currently under fabrication.

\section{LITERATURE}

[1] Randall K. Kirschmann, Cold electronics: An Overview, Cryogenics,vol. 25 1985,p.115-121

[2] Ottmar Kindl, Tiefsttemperatur CMOS Technik für Anwendungen in der Infrarotastronomie, VDI-Verlag Düsseldorf, 1989

[3] S. Schwehr, W. Langheinrich, Using the Subthreshold Characteristic for Low-Noise, LowPower Operational Amplifiers at $4.2 \mathrm{~K}$, Proceedings of the Symposium on Low Temperature Electronics and High Temperature Superconductivity, The Electrochemical Society, Hawaii 1993

[4] P. P. Debye, E. M. Conwell, Physical Review 93,1954, p. 693

[5] J. A.del Alamo, Minority carrier transport in heavily doped n-type silicon,Stanford SEL Tech. Rep. 1984

[6] J. Vollrath, W. Langheinrich, A CMOS compatible JFET Technology for Operation down to $4.2 \mathrm{~K}$, Proceedings of the Symposium on Low Temperature Electronics and High Temperature Superconductivity, The Electrochemical Society, Hawaii 199:3

[7] B. Lengeler, Semiconductor Devices Suitable for Use in Cryogenic Environments, Cryogenics, 1974, p.439-447

[8] J. M. Dorkel, Ph. Leturcq, Carrier Mobilities in Silicon Semi-empirically related to temperature, doping and injection level, Solid State Electronics,vol. 24, 1981,p.821-825 